\title{
Acute Undifferentiated Leukemia
}

National Cancer Institute

\section{Source}

National Cancer Institute. Acute Undifferentiated Leukemia. NCI Thesaurus. Code C9298.

A rare acute leukemia of ambiguous lineage in which the blasts do not express markers specific to myeloid or lymphoid lineage. 